\title{
Simulation of Wave motion using a Lattice Gas Model
}

\author{
J. Buick ${ }^{\dagger}$ \\ W. Easson ${ }^{\ddagger}$ \\ C. Greated ${ }^{\dagger}$ \\ $\dagger$ Department of Physics \\ The University of Edinburgh \\ James Clerk Maxwell Building \\ The King's Buildings \\ Mayfield Road \\ Edinburgh \\ EH9 3.JZ \\ †Department of Mechanical Engineering \\ The University of Edinburgh
}




\begin{abstract}
The lattice gas model for simulating two phase flow, proposed by Appert and Zaleski, has been modified by the introduction of gravitational interactions and the new model has been used to simulate standing wave patterns on the free surface of a fluid. The results compare well with linear theory.
\end{abstract}

Key words: Lattice gas, Cellular automata, Wave Modelling, Standing waves.

\title{
1 Introduction
}

\subsection{Lattice gas modelling}

In recent years lattice gas models have been used to model a variety of fluid phenomena such as flow round plates [1], and also more complicated simulations involving two or more fluids such as the Kelvin-Helmholtz instability [2] and the combustion of two gases to produce a third [3].

Although less well developed than techniques such as finite difference and finite element solutions of the Navier-Stokes equation, the lattice gas approach has inherent advantages in the solution of complex phenomena such as wave motion through media with density gradients and in simulations where mixing or phase changes may occur.

The lattice gas model was first introduced by Frisch, Hasslacher and Pomeau (FHP) [4]. Their formulation is the basis for the model used in our simulations and is described below. The FHP model consists of an ensemble of fluid 'particles' which move on an underlying hexagonal grid. Each particle moves along one of the six link directions $d_{\alpha}(\alpha=1,6)$ where the direction of $d_{\alpha}$ is given by $\cos \left(\frac{\pi \alpha}{3}-\frac{\pi}{6}\right) \underset{\sim}{i}+\sin \left(\frac{\pi \alpha}{3}-\frac{\pi}{6}\right) \underset{\sim}{j}$, as shown in figure 1 . The particles travel at unit speed, moving from one intersection point, or site, to a neighbouring site in each time step. Only one particle can travel along any one link at any time. At the end of each time step all the particles coming into a particular site are allowed to collide in such a way that the momentum and number of particles at that site are conserved. Particles then move off in their new directions at the beginning of the next time step. The general lattice gas model can be expanded to allow any number of rest particles to be present at each of the sites $\left(d_{0}\right)$. In the following simulations the FHPIII model is used, which allows a maximum of one rest particle at each site.

The basic FHP collisions [5] are shown in figure 2, where the left hand column shows the incoming configurations, and the right hand column shows the possible outcomes. Rest particles are represented by a solid sphere. When there is more than one possible outcome, one of these is selected at random. The FHPIII collision rules are formed from the basic collisions, the basic collisions with a 
spectator particle (moving or at rest), and the dual of these collisions formed by swapping particles and empty links. Free-slip conditions can be produced at a solid boundary by flipping all incoming particles in such a way that the particle momentum parallel to the boundary is conserved and the particle momentum perpendicular to the boundary is reversed [6]. Thus a particle approaching a horizontal free-slip boundary along link $d_{6}$, see figure 1, (travelling towards the site) will leave the site travelling along link $d_{1}$. Macroscopic fluid quantities such as velocity and density can be found by averaging the microscopic quantities over a cell, typically no smaller than 16 sites by 16 sites [5].

It can be shown $[5,4]$ that for the FHPIII model the macroscopic collision rules satisfy the equations

$$
\partial_{t} \rho+\sum_{\alpha} \partial_{\alpha}\left(\rho u_{\alpha}\right)=0
$$

and

$$
\partial_{t}\left(\rho u_{\alpha}\right)+\sum_{\beta} \partial_{x_{\beta}}\left[\rho g(d) u_{\alpha} u_{\beta}\right]=-\partial_{x_{\alpha}} P+\sum_{\beta} \partial_{x_{\beta}}\left[\nu(\rho) \partial_{\beta} u_{\alpha}\right]
$$

where $\rho$ is the density, $P$ is the pressure, $d$ is the density per link, $d=\rho / 7, \nu$ is the bulk viscosity and $g(d)=\frac{7(1-2 d)}{12(1-d)}$. These are the continuity equation and the Navier-Stokes equation with an extra factor $g$. The Navier-Stokes equation can be recovered by rescaling time, pressure and viscosity by $g: t^{\prime} \rightarrow t g, \quad P^{\prime} \rightarrow P / g$, $\nu^{\prime} \rightarrow \nu / g$.

\subsection{Gravitational Interactions}

Gravitational interactions can be simulated by flipping a small number of particles after the collision from link $d_{1}$ to $d_{3}$ and from link $d_{6}$ to $d_{4}$, provided there is not already a particle travelling in that direction. This interaction was introduced into the FHP model by allowing particles to be flipped every time step. The gravitational interaction is performed after the particles have collided and has the effect of decreasing the momentum in the y-direction while leaving the momentum in the $\mathrm{x}$-direction unchanged. This interaction produces a density gradient across the fluid; however this will not be particularly large provided the number of gravitational interactions is small [6]. Here we restrict the number of such flips to be a half percent of the total number of possible flips. This gives an average of $2.5 \times 10^{-3}$ flips per site per time step. This is significantly smaller than the number of FHP collisions taking place: in a 25,000 time step simulation there will only be on average 62.5 gravity flips performed at each site compared to an average of 15,000 FHP collisions [1]. 


\subsection{Liquid-Gas Model}

A liquid-gas (LG) model is a lattice gas model which undergoes a phase-separation producing a separate light and dense phase. This phase-separation is produced using interaction rules which were first introduced by Appert and Zaleski [7, 8] and are shown in figure 3. The interaction operates on two sites a distance IL apart in either of the directions $d_{1}, d_{2}$ and $d_{3}$ (figure 3 shows the interaction rules acting in direction $d_{2}$ ). Particles travelling along the links represented by the solid arrows are flipped into the directions shown by the dotted arrows provided there are particles in both the initial links and no particles in either of the destination links. These interaction rules clearly conserve overall momentum, although they do not conserve the momentum at each site. The value of IL used in the following simulations was 3 lattice units.

\subsection{Implementation of the model}

The model was implemented on the Connection Machine (CM) 200 at The University of Edinburgh, the different interactions described above being implemented in the following order:

\section{FHP III collision rules}

2. Gravitational interactions

3. Interaction(a) in direction $\mathrm{i}$, direction ii, then direction iii

4. Interaction(b) in direction $\mathrm{i}$, direction ii, then direction iii

5. Interaction(c) in direction $\mathrm{i}$, direction ii, then direction iii

6. Interactions (d) and (e) combined in direction i, direction ii, then direction iii,

where directions $\mathrm{i}$, ii and iii are a random ordering of $d_{1}, d_{2}$ and $d_{3}$, the ordering being different for each of the four implementations $(3)-(6)$ above. Interactions (d) and (e) are combined to prevent a net clockwise or anti-clockwise rotation being imposed on the fluid. 


\subsection{Numerical Modelling}

One of the main advantages of the FHP model is its simplicity:

- The state of the particles on the lattice need only be known at discrete time intervals. There is no need to track the particles when they are moving from one site to a neighbouring site.

- The state of each site at any time step can be expressed as a 7-bit integer, one bit for each link taking the value 1 or 0 in the presence or absence of a particle on the link.

- The FHP collisions can be implemented using a look-up table taking the initial particle configuration at the site (an integer in the range 0 - 127) and returning the new configuration after the collision. This removes the need to calculate the outcome of each collision.

- Updating the lattice between time steps requires only a knowledge of the particle states at each of the six neighbouring sites. This is done by shifting the lattice in each of the six link directions.

When introducing the new interactions it is important to try and keep the underlying simplicity of the FHP model. The gravitational interaction is performed by first selecting all the sites where the interaction can take place and then selecting a percentage (we used $0.5 \%$ ) of these at which the interaction is allowed to take place. The interaction can be modelled using simple addition and subtraction: a particle being flipped from link 1 (integer value $2^{1}$ ) to link 3 (integer value $2^{3}$ ) results in $2^{3}-2^{1}$ being added to the integer representing the particles at the site. In practice it was found best to consider the two possible gravity flips $\left(d_{1} \rightarrow d_{3}\right.$ and $\left.d_{6} \rightarrow d_{4}\right)$ separately.

In principle the Appert and Zaleski interactions can be implemented simply by first shifting the world $I L$ units in the chosen direction and comparing the shifted configuration with the original configuration to see if an interaction can take place. If an interaction can take place this is done, again using simple arithmetic, to the shifted lattice. Finally the grid is shifted back to its original position and the inverse arithmetic operation applied. Thus for example if interaction (a) of figure 3 is to be applied to the sites $(1,1)$ and $(1,4)$ then site $(1,1)$ is first shifted three units to the left to position $(1,4)$. The two grids are then considered at the point $(1,4)$ to see if the interaction can take place. This can only happen if bit5 $=1$ and bit $2=0$ on the shifted grid and bit $5=0$ and bit $2=1$ on the original grid. The integer $\left(2^{5}-2^{2}\right)$ is then subtracted from the integer representation at 
the point $(1,4)$ on the shifted grid. The altered point $(1,4)$ is then shifted back to its original position $(1,1)$ and finally $\left(2^{5}-2^{2}\right)$ must be added to the integer representing the point $(1,4)$. This is done in parallel to all points on the grid at the same time. If no interaction can take place the integers at the points $(1,1)$ and $(1,4)$ remain unchanged. They may however be changed by on eof the subsequent interactions.

The implementation of this interaction is however complicated slightly by the need to randomise the order in which the interactions (a) - (e) are performed and also the order in which each interaction is performed along the three possible directions.

\section{Standing Wave Simulations}

The modified LG model was used to simulate standing waves on a 4096 x 256 grid. Continuous boundary conditions were used, with a solid free-slip boundary placed at the bottom of the grid. Initially a standing wave was set up with wavelength 4096 units and depth 180 units. This was done by first allowing the fluid to settle, with a horizontal interface between the two phases at the mean water level (mwl). Area A, shown in figure 4, was then filled with stationary fluid of the same density as the fluid just below the mwl, and areas B were filled with stationary fluid of the same density as the fluid just above the mwl. The standing wave was then allowed to oscillate under the effect of the gravitational interactions. This was repeated six times, starting from the same initial conditions but using a different set of random numbers during the simulations. The velocity results shown below are averaged over the six simulations.

\section{Results and Discussion}

The wave height at its centre was measured every forty time-steps; this is shown in figure 5 for one of the six simulations along with the best fit curve of the form $A e^{-\alpha t} \cos \left(\frac{2 \pi t}{\tau}+\psi\right)+h$, where $A=15.0, \alpha=5.16 \times 10^{-5}, \tau=10,100, \psi=$ $-0.139, h=165$, all in units of time-steps and lattice lengths. Although it is possible to initialise a wave with a density gradient which is a good approximation to its natural gradient, there will always be a short initial period during which the wave re-adjusts itself. The variable $\psi$ is added to account for this and to allow for the wave not starting to oscillate at $t=0$ from exactly the start of a period. It can be seen from the best fit data that $\psi$ is equivalent to $\sim 200$ time-steps. The velocity field under the standing wave at $t=3,000$ time-steps is shown in figure $6 \mathrm{a}$ and can be compared to figure $6 \mathrm{~b}$ which shows the corresponding velocities computed for a wave with the same depth-to-wavelength ratio, at time 
$t=\tau / 4$ using the linear wave theory equations [9]

$$
\begin{aligned}
& v_{x}=\frac{-a \omega \cosh (y+h)}{\sinh (k h)} \sin (k x) \sin (\omega t) \\
& v_{y}=\frac{a \omega \sinh (y+h)}{\sinh (k h)} \cos (k x) \sin (\omega t),
\end{aligned}
$$

where $h$ is the mean water depth and $y$ is measured vertically upwards from the mean free surface. The plots in figure 6 a are in lattice units; the plots in figure $6 \mathrm{~b}$ have been normalised so that the peak $\mathrm{x}$-velocity at the surface correspond in magnitude to that found from the model. The linear theory y-velocities were normalised by the same factor. Figure 6.1 shows a vector plot of the velocities, figure 6.2 the $\mathrm{x}$-velocity profile across the phase of the wave at different heights $(y)$, figure 6.3 shows a contour plot of the $\mathrm{x}$-velocity and figure 6.4 shows the y-velocity profile. In figures $6.2 \mathrm{a}$ and $6.4 \mathrm{a}$ the symbols are the experimental data points and the solid curves are produced by filtering out the noise with a Fourier filter. It can be seen from figures $6.2 \mathrm{a}$ and $6.4 \mathrm{a}$ that the $\mathrm{y}$-velocities are significantly smaller than the x-velocities and the signal-to-noise ratio for the $y$ velocity is small. This causes a contour plot of the $y$-velocities to be particularly noisy and of little interest.

The results obtained compare well with the linear theory, except for two obvious differences. Firstly the x-velocity decreases with the distance below the surface faster than predicted by the theory; this can be seen best when comparing figures $6.2 \mathrm{a}$ and $6.2 \mathrm{~b}$. Secondly the ratio of $\mathrm{x}$-velocity : y-velocity is slightly larger than is predicted by linear theory. The $\mathrm{x}$-velocities from linear theory were scaled so that their magnitude would be as close as possible to the computed x-velocities; when the $y$-velocities are scaled by the same factor the computed $y$-velocities are found to be smaller by a factor $\sim 0.8$. Both of these differences are consistent with the simulated wave having an altered depth-to-wavelength ratio. The main cause of this effect is found to be caused by the density gradient which is produced across the fluid by the gravitational interactions. This change in density with depth which is not present in the linear theory is seen to have the effect of changing the apparent depth of the fluid. The ratio of the real depth and the apparent depth can be accounted for in any simulation since it is found to depend solely on the strength of the gravitational interaction. The strength of the gravitational interaction used here has the effect of causing the fluid to appear approximately twice its actual depth. Another factor which changes the depth of the fluid is the fact that the long range interactions do not act at the bottom boundary. This effects the density of the fluid at the bottom three or four sites which will change the effective depth only slightly, but significantly since we are dealing with shallow water waves. It is necessary that the long range interaction does not act at the boundary to prevent particles becoming trapped in the boundary.

To investigate the effect of grid size on the model another wave was initialised on 
a 2048 by 128 grid with a wave of wavelength 2048 lattice units and a mwl of 91 lattice unit. The period of this wave was found to be 7423 time-steps. This wave has half the wavelength and approximately half the depth of the wave already discussed. We can now compare the Reynolds numbers of the two waves: the Reynolds number is given by

$$
R e=\frac{L U g(d)}{\nu}
$$

where $L$ and $U$ are characteristic lengths and velocities respectively of the simulation and $\nu / g(d)$ is the scaled viscosity, see section 1.1, which will be the same for both waves since the density is the same. In this comparison we will use a subscript ' 1 ' for the shorter wave and a subscript ' 2 ' for the longer wave. For surface waves Froude scaling applies with $\tau_{2} / \tau_{1}=\sqrt{\lambda_{2} / \lambda_{1}}$ : in this case $\tau_{2}=\sqrt{2} \tau_{1}$, $[10]$.

Comparing the ratio of the periods of the two waves we see that $\tau_{1} / \tau_{2}=1.46$ $\simeq \sqrt{2}$ to within $3 \%$. The characteristic velocity is taken to be the peak $\mathrm{x}$-velocity of an undamped wave. This is given by

$$
U=v_{x}(x=\lambda / 4, y=0, t=\tau / 4) \exp \{\alpha \tau / 4\} .
$$

The factor $\exp \{\alpha \tau / 4\}$ compensates for the damping which occurs during the first quarter period and is required because the velocity is damped at different rates for different waves. Using this characteristic velocity we get $U_{1} / U_{2}=0.75 \simeq \sqrt{1 / 2}$ to within $6 \%$ which is as expected since $U_{i} \propto \tau_{i}^{-1}$. Thus comparing Reynolds numbers $R e_{1}=R e_{2}$ we see that doubling the size of the wave has reduced the effective viscosity by a factor of $\sqrt{2}$.

A calculation of the Reynolds number requires the value of the scaling constant $g(d)$ and of the viscosity $\nu$. These have been found experimentally however their measurement is beyond the scope of this paper. The value obtained for $g(d)$ and $\nu$ are 0.6 and 20 respectively where both values are in lattice units. This gives a Reynolds number $\sim 10$. The high viscosity present in the model is the main restraint on the Reynolds number which can be achieved. This is a common feature of lattice gas simulations which is increased in this model by the introduction of the additional interactions.

\section{Conclusion}

The close comparison between the results obtained and the linear theory suggests that a LG model is suitable for modelling surface waves. The waves produced are clearly highly viscous, which is one of the features of a lattice gas model. The viscous effect can be reduced by using a larger grid and increasing the wavelength of the wave. It may also be possible to alter the fluid density by changing the long range interaction rules which would change the viscosity of the model. 


\section{Acknowledgements}

The authors would like to acknowledge the assistance of Paul Stansell who coded an original version of the program which was used in the simulations. The first author would like to acknowledge the support of the Science and Engineering Research Council.

\section{References}

[1] D. d' Humières and P. Lallemand. Numerical simulations of hydrodynamics with lattice gas automata in two dimensions. Complex Systems, 1:599, 1987.

[2] D. d' Humières, P. Lallemand, and G. Searby. Numerical experiments on lattice gasses: Mixtures and galilean invariance. Complex Systems, 1:633, 1987.

[3] G. Searby, V Zenlé, and B Denet. Lattice gas mixtures and reactive flows. In Discrete Kinetic Theory, Lattice Gas Dynamics and the Foundations of Hydrodynamics, Torino, Italy, September 1988.

[4] U. Frisch, B. Hasslacher, and Y. Pomeau. Lattice-gas automata for the navier-stokes equation. Physical Review Letters, 56:1505, 1986.

[5] U. Frisch, D. d' Humières, B. Hasslacher, P. lallemand, Y. Pomeau, and J-P Rivet. Lattice gas hydrodynamics in two and three dimensions. Complex Systems, 1:649, 1987.

[6] J. P. Boon and A. Noullez. Lattice gas hydrodynamics. In Special Course on Modern Theoretical and Experimental Approaches to Turbulent Flow Structure and its Modelling. AGARD (Advisory Group for Aerospace Research and Development) NATO, 1987.

[7] C. Appert and S. Zaleski. Lattice gas with a liquid-gas transition. Physical Review Letters, 64:1, 1990.

[8] C. Appert, D. Rothman, and S. Zaleski. A liquid-gas model on a lattice. Physica D, 47:85, 1991.

[9] G. D. Crapper. Introduction to Water Waves. Ellis Horwood Limited, 1984.

[10] B. Le Méhauté. An Introduction to Hydrodynamics and Water Waves. Springer-Verlag, 1976. 


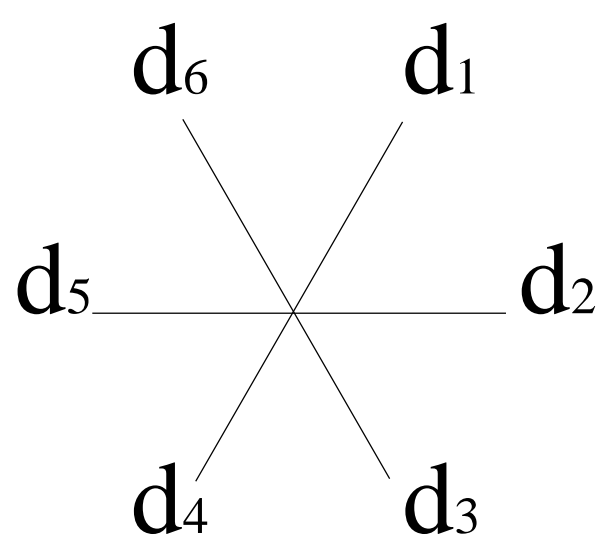

Figure 1: The six directions of the hexagonal lattice. 

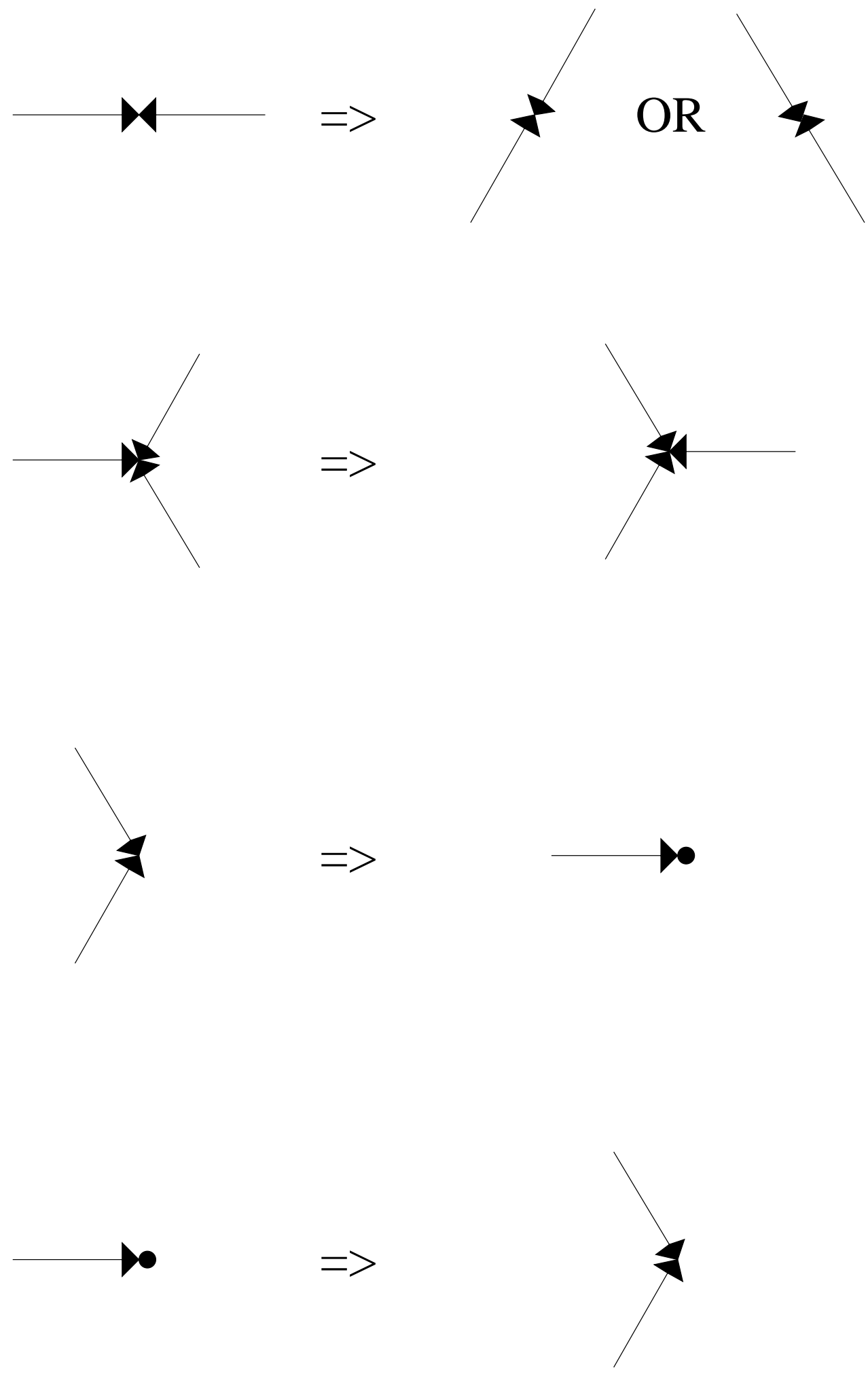

Figure 2: The basic FHP collision rules. 

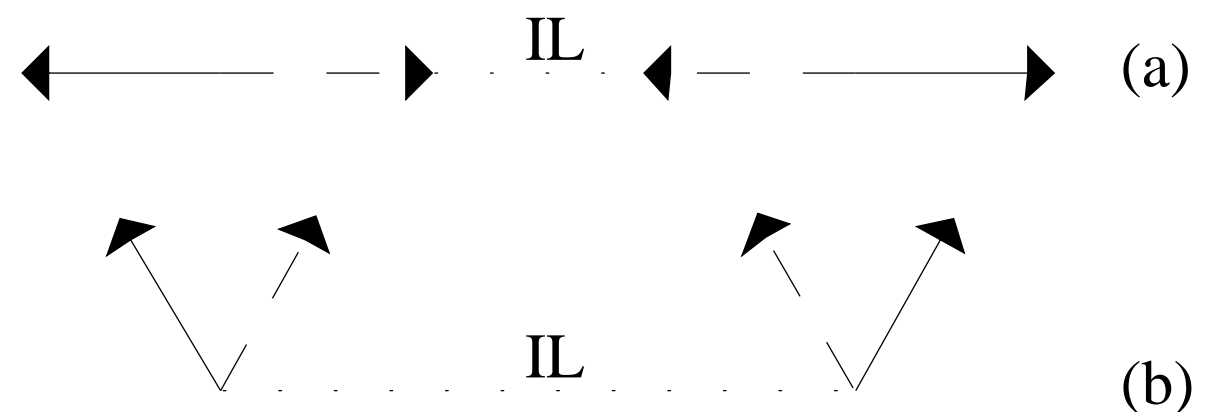

IL
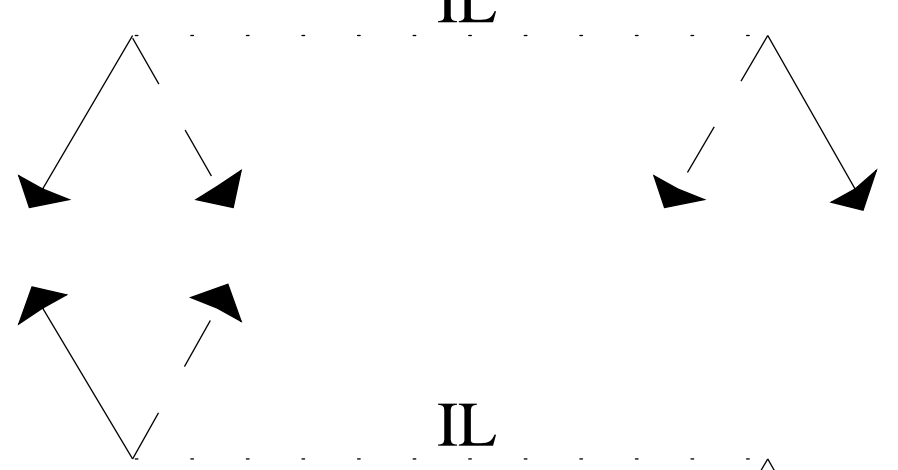

IL
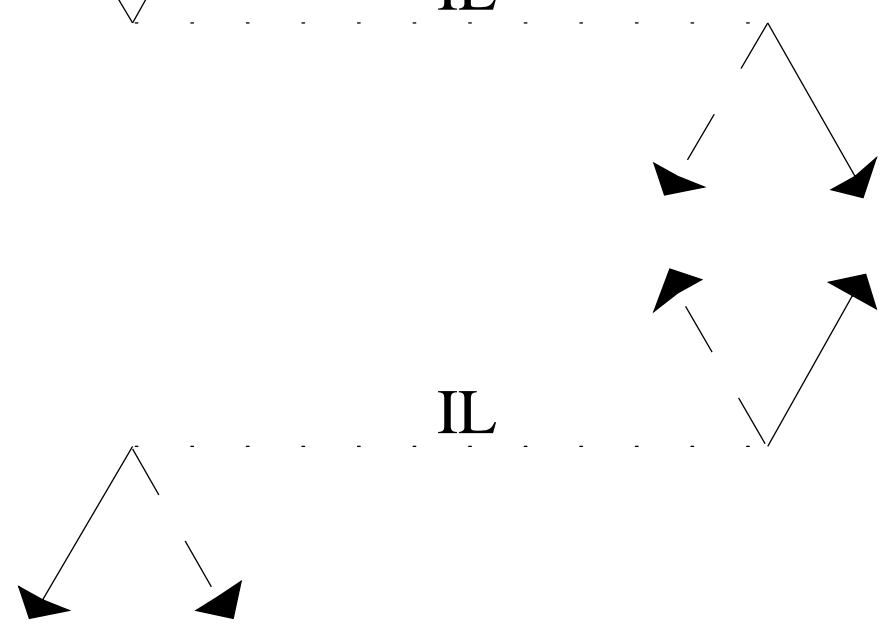

(b)

(c)

(d)

Figure 3: The long range interaction. 


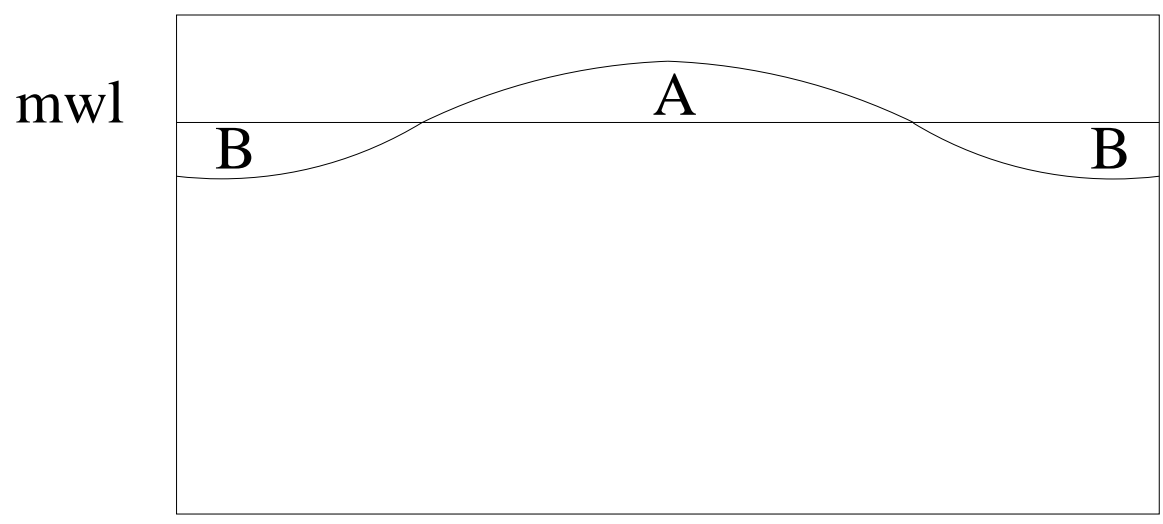

Figure 4: The construction of the standing wave from a flat surface. Note that figure 4 is not draw to scale. The wave is in fact very shallow with an amplitude of $\sim 15$ units and a wavelength of 4096 units. 


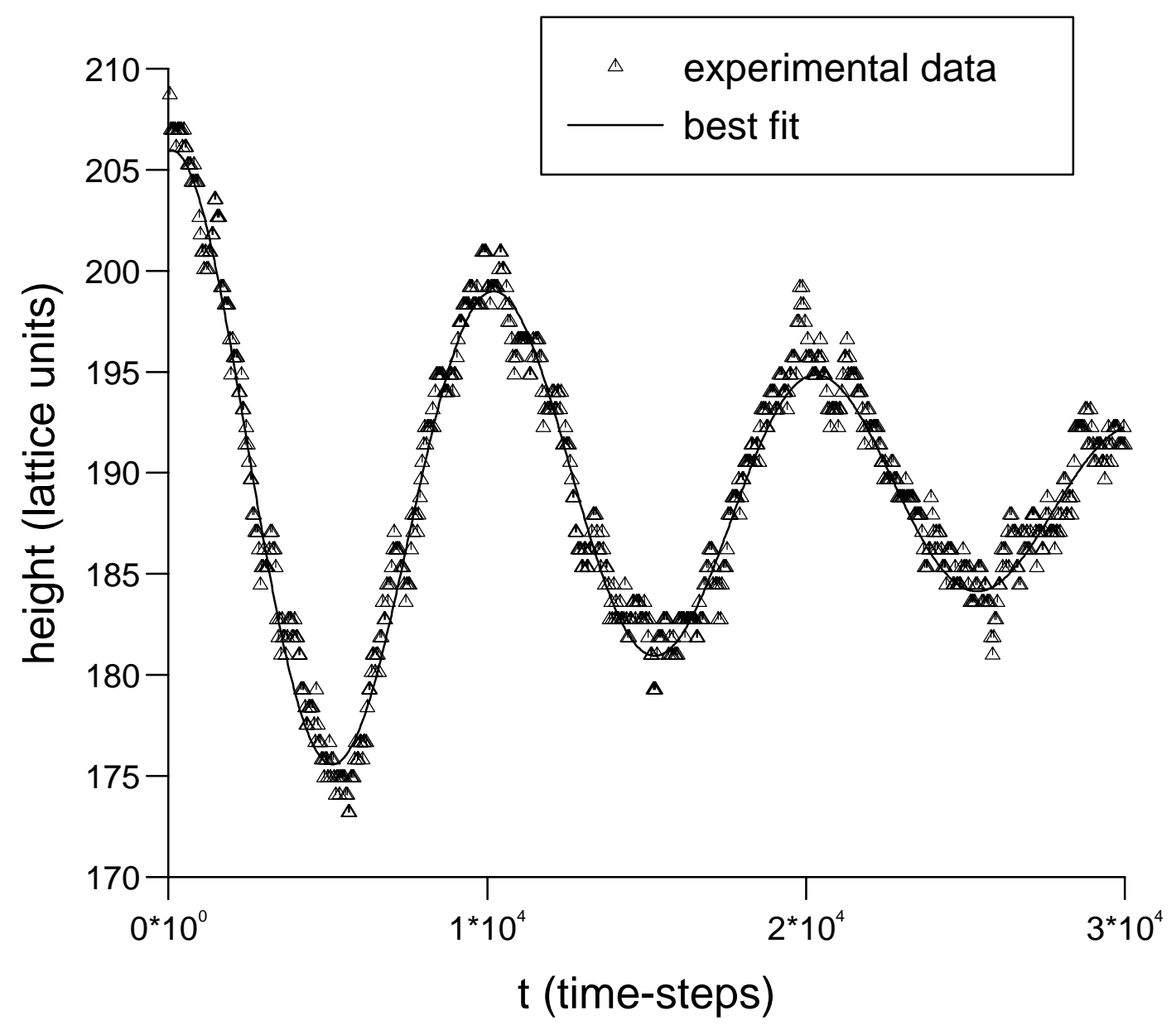

Figure 5: The height of the wave centre for one of the six runs plotted against time and the best fit curve. 


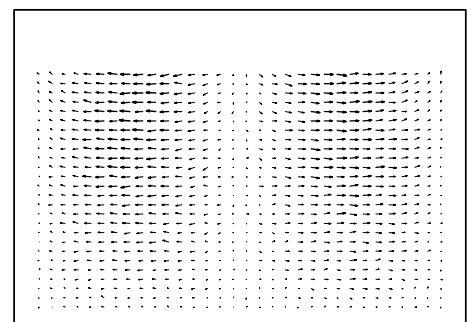

1a: velocity vector plot

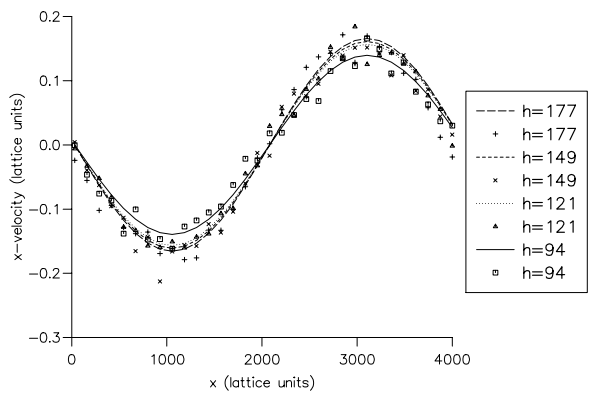

2a: $\mathrm{x}$-velocity profile

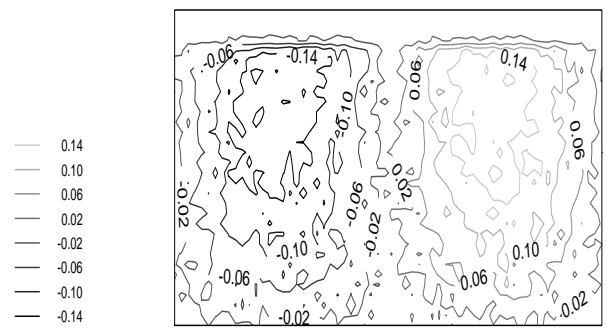

3a: $\mathrm{x}$-velocity contour plot

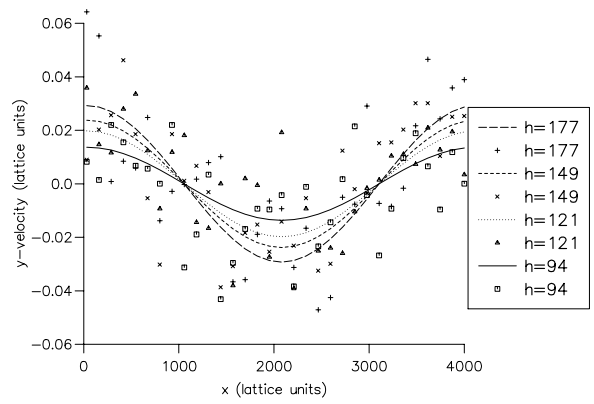

4a: y-velocity profile

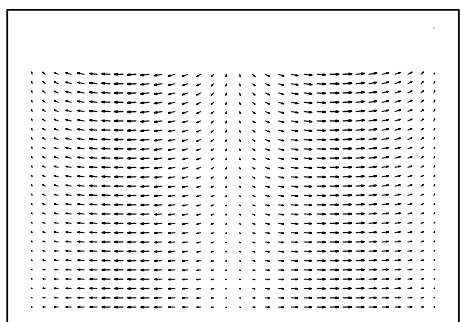

$1 \mathrm{~b}:$ velocity vector plot

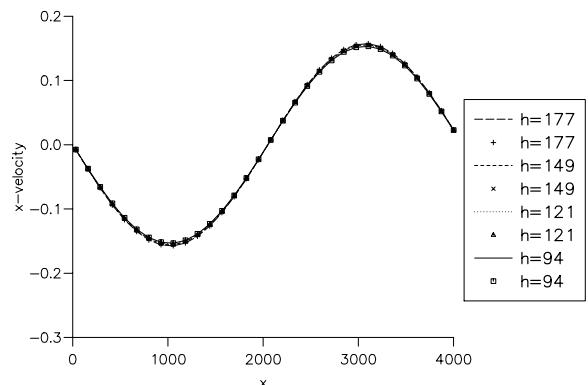

2b: $\mathrm{x}$-velocity profile

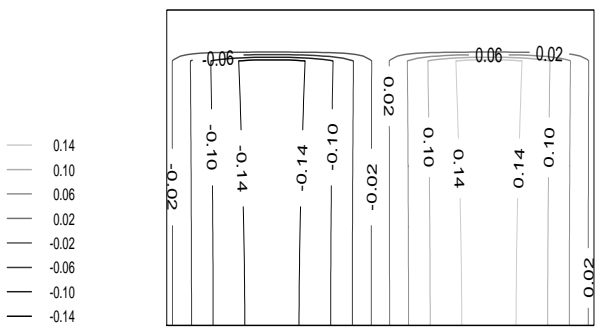

3b: x-velocity contour map

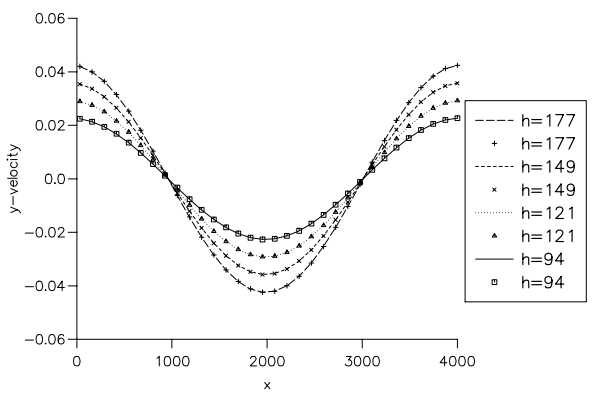

4b: y-velocity profile

Figure 6: The wave velocities at $\mathrm{t}=3,000$ time-steps 